\title{
Structural Integration of Ku-band SatCom Antenna into novel Fuselage Panel
}

\section{J. VERPOORTE, A. HULZINGA}

Netherlands Aerospace Centre NLR

Voorsterweg 31, 8316 PR Marknesse, The Netherlands

e-mail: jaco.verpoorte@nlr.nl, adriaan.hulzinga@nlr.nl

Key words: Antenna, integration, lightning protection, Ku-band, satellite communication, orthogrid.

\begin{abstract}
This paper addresses the structural integration of a Ku-band SATCOM antenna in the fuselage of an aircraft. The phased array antenna consists of 25 antenna tiles that are integrated in an orthogrid fuselage panel. The specific electromagnetic aspects of the antenna integration will be discussed in this paper; this concerns the lightning protection of the antenna tiles and the electromagnetic interaction of the CFRP orthogrid and GFRP fuselage skin with the array antenna. The structural properties and thermal management of the antenna will be discussed in separate papers $[1,2,3]$.
\end{abstract}

\section{INTRODUCTION}

The main objective of the ACASIAS project is to contribute to the reduction of energy consumption of future aircraft by improving the aerodynamic performance through conformal and structural integration of antennas that are normally protruding. This paper deals with the conformal integration of an electronically steerable Ku-band antenna for satellite communication, which does no longer require a protruding radome.

In the ACASIAS project a composite stiffened orthogrid fuselage panel is being developed for integration of Ku-band SATCOM phased array antenna tiles (Figure 1). The Ku-band antenna tiles to be integrated are based on the antenna tiles that were developed in a previous FP7 project called SANDRA. In the SANDRA project the focus was on the functional performance of the antenna tiles. In the ACASIAS project the focus will be on the integration of the antenna tiles in a fuselage panel, taking into account integration aspects like thermal control and lightning protection. The ribs of the orthogrid will be made of Carbon Fibre Reinforced Plastic (CFRP), while the skin will consist partly of CFRP and partly of Glass Fibre Reinforced Plastic (GFRP). The GFRP skin is necessary to enable the electromagnetic radiation from the antenna underneath it. The orthogrid has a pitch of $107 \mathrm{~mm}$. The rib thickness is $7 \mathrm{~mm}$ and the rib height is $8 \mathrm{~mm}$. The array consists of 5 by 5 antenna tiles. Each antenna tile has 8 by 8 antenna elements. The tile will have a maximum size of 100 by $100 \mathrm{~mm}$ and a thickness of $7.8 \mathrm{~mm}$. The tiles may be made a bit smaller to accommodate tolerances and deformation of the orthogrid.

The structural design and analysis of the orthogrid will be discussed in separate papers $[1,2]$. 


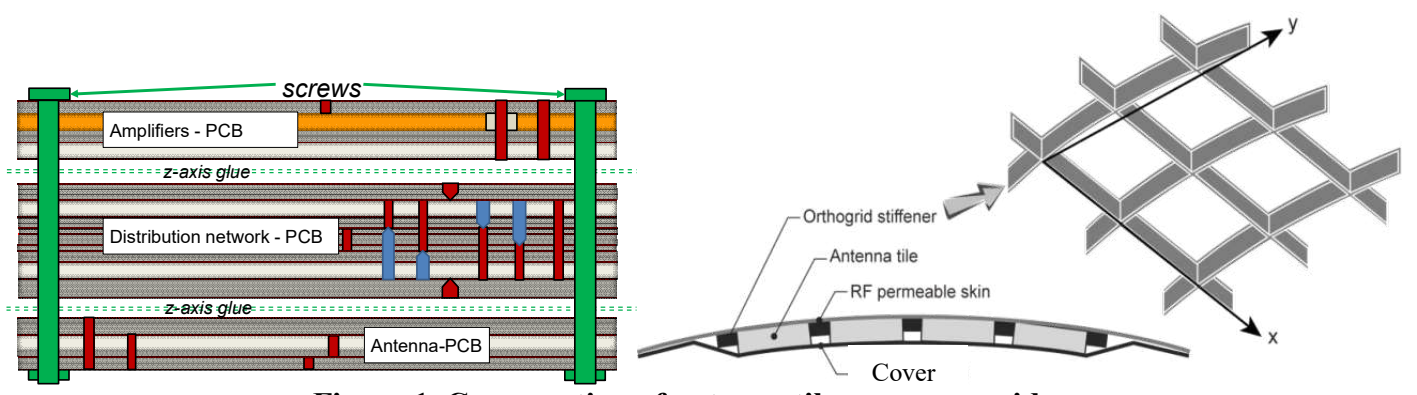

Figure 1. Cross section of antenna tile and orthogrid.

A complete array of 25 antenna tiles will be designed and manufactured. However, not all tiles will be real antennas since the main focus in this project is on cooling solutions and lightning protection. Some tiles will only have passive components (resistors) and will not operate as an antenna. The thermal behaviour of these dummy tiles will be representative for the real antenna tiles. The antenna tiles will have built-in cooling solutions. These cooling solutions and the thermal behaviour of the antenna tiles will be discussed in a separate paper [3]. This paper will focus on the electromagnetic aspects of the antenna integration: the influence of the fuselage structure and of the lightning protection material on the behaviour of the satcom antenna.
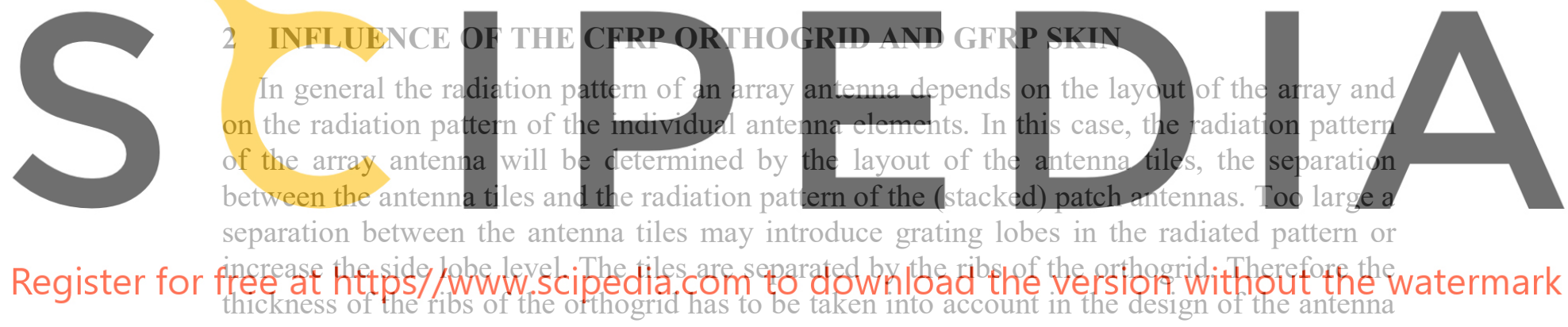

array. In addition, the influence of the material of the ribs on the radiation pattern has to be taken into account. The CFRP ribs of the orthogrid are conductive. In the design of the antenna elements and antenna tiles the conductivity of the ribs will have to be taken into account, especially under scanning conditions of the antenna. The skin of the fuselage panel above the antenna tiles is transparent to enable the electromagnetic radiation by the antenna. Therefore the complex permittivity of the GFRP material will have to be determined to assess the specific transmission, reflection and absorption properties of the GFRP skin for Ku-band waves.

\subsection{Influence of tile separation on radiation pattern}

The antenna tiles in the orthogrid are separated by the ribs of the orthogrid. In order to obtain sufficient structural strength, the ribs of the orthogrid have a minimum width $(7 \mathrm{~mm})$ and height $(8 \mathrm{~mm})$. Due to the rib thickness the antenna tiles do not touch but have a separation of $7 \mathrm{~mm}$. This is in the order of a quarter of the wavelength and therefore this gap will have an influence on the radiation pattern. In Figure 2 the influence is shown of the gap between the antenna tiles. When the gap increases from 0 to $3 \mathrm{~mm}$ some staircasing becomes 
visible in the radiation pattern. This implies that the sidelobe levels increase slightly at some angles. When the gap increases from $3 \mathrm{~mm}$ to $7 \mathrm{~mm}$ the sidelobes increase even more. Recommendations are given for the radiation pattern of transmit antennas for Ku-band satellite communication [4,5]. In order to meet these standards, the sidelobes have to be suppressed to a certain level. This can be done by applying an amplitude taper over the array. However, this will reduce the gain and broaden the main lobe also. In addition it adds to the system complexity because not only the phase of the antenna elements has to be controlled (for beam steering) but also the amplitude (for side level reduction). As an example, the effect of applying a Chebyshev tapering is shown in Figure 3.

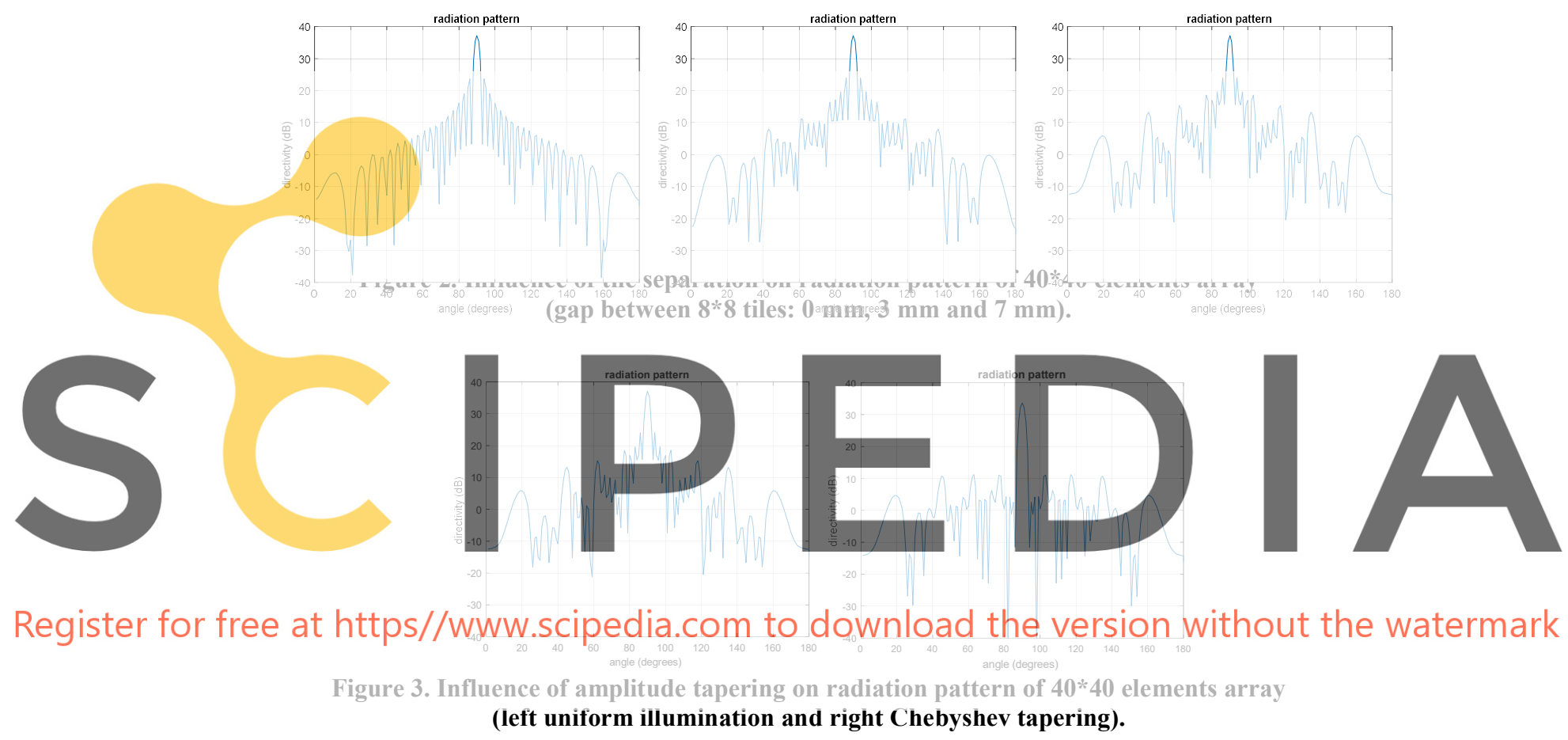

\subsection{Influence of CFRP orthogrid on the radiation pattern of antenna tile}

The ribs are made of CFRP. This material has a specific conductivity and will therefore have an influence on the input impedance and the radiation pattern. Since the ribs are at the edge of an $8^{*} 8$ array, the effect will be mainly on the edge elements and less on the centre elements. The antenna tiles will be installed in the orthogrid with a certain air gap between the antenna tile and the skin to accommodate for deformation of the fuselage panel and to decrease the influence of the skin on the antenna performance. In Figure 5 the influence on the radiation pattern for two orthogonal polarisations is given as a function of the height of the rib. The radiation pattern is given for three different rib heights $(0,3$ and $6 \mathrm{~mm})$, where the height given is the height of the rib above the antenna tiles (the actual height of the ribs is $8 \mathrm{~mm}$ ). In Figure 5 the radiation pattern for a corner element of an antenna tile is shown (X11) and in Figure 6 the radiation pattern is given of the second antenna element (X22) along the 
diagonal of the antenna tile (Figure 4 shows the position of the antenna elements).

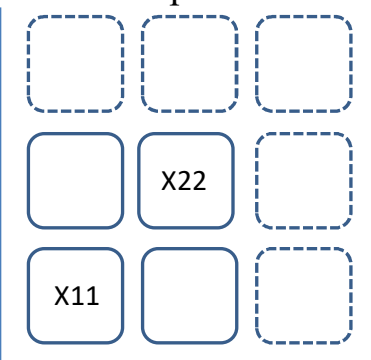

Figure 4. Numbering of antenna elements in array.

The radiation pattern is given for a single antenna element in a small array (4 by 4 ), therefore this antenna also radiates to the back. The influences of the rib on the forward radiation of the antenna is very small. Some influence can be seen on the backward radiation of the antenna element. In general the influence is larger when the rib height is larger. The influence is most significant for the corner element (X11) and less for elements more to the centre (like X22).
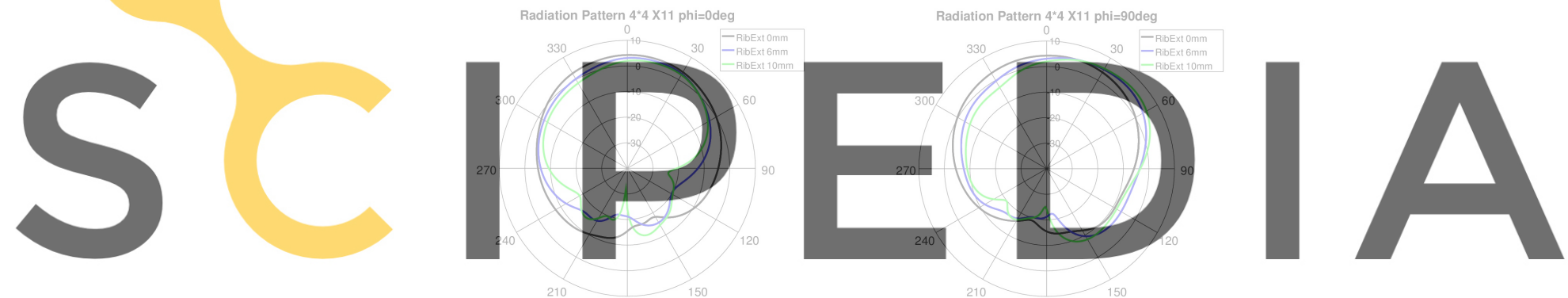

Register for free at https//www.scipedia.com to download the version without the watermark Figure 5. Influence of rib height on the radiation pattern of an antenna element in the corner of the antenna tile X11 (two orthogonal polarisations).
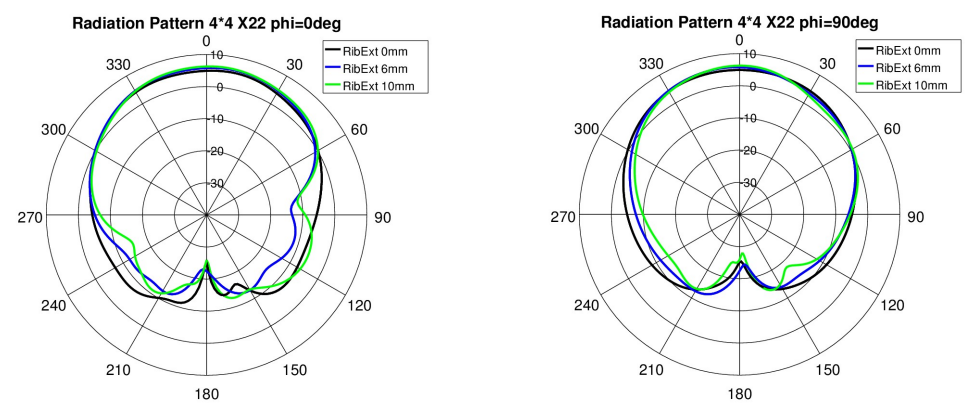

Figure 6. Influence of rib height on the radiation pattern of an antenna element near the corner of the antenna tile X22 (two orthogonal polarisations). 


\subsection{Influence of the GFRP skin on the input impedance and radiation pattern of the antenna}

In Figure 7 the influence of the thickness of and the distance to the GFRP skin is given. The skin is made of S-2 glass fibres. In this case the influence of the skin on the reflection loss $\left(\mathrm{S}_{11}\right)$ of a single antenna element is given. The antenna element is designed to give a reflection loss less than $-10 \mathrm{~dB}$ between $10.7 \mathrm{GHz}$ and $12.7 \mathrm{GHz}$. If the skin has a thickness of $1 \mathrm{~mm}$ the $\mathrm{S}_{11}$ increases to about $-6 \mathrm{~dB}$, which is too high. For larger skin thickness (e.g. $6 \mathrm{~mm}$ ) the $\mathrm{S}_{11}$ shows a better behaviour: the reflection loss is below $-10 \mathrm{~dB}$ in a larger part of the frequency band. Probably a skin with a thickness of $6 \mathrm{~mm}$ resembles a radome with thickness of half a wavelength which has in general good transmission and reflection characteristics. A skin with a thickness of much less than $1 \mathrm{~mm}$ will probably also give a good performance. If a skin of $6 \mathrm{~mm}$ is too heavy and a skin of less than $1 \mathrm{~mm}$ is too thin from a structural point of view, then a sandwich construction could be applied (two thin layers of S-2 glass with a low permittivity material in between having a total thickness of about $6 \mathrm{~mm}$ ). In the structural design, the thickness of the GFRP layer is currently fixed at $2.1 \mathrm{~mm}$. If the layer is thicker, the glass window will become too stiff and will absorb all the forces in the fuselage panel. To obtain a better $\mathrm{S}_{11}$ with a $2.1 \mathrm{~mm}$ GFRP skin, material with same permittivity as glass has to be applied between the skin and the antenna tile to get a good performance. Also the design of the antenna element needs to be optimised for the presence of the GFRP skin
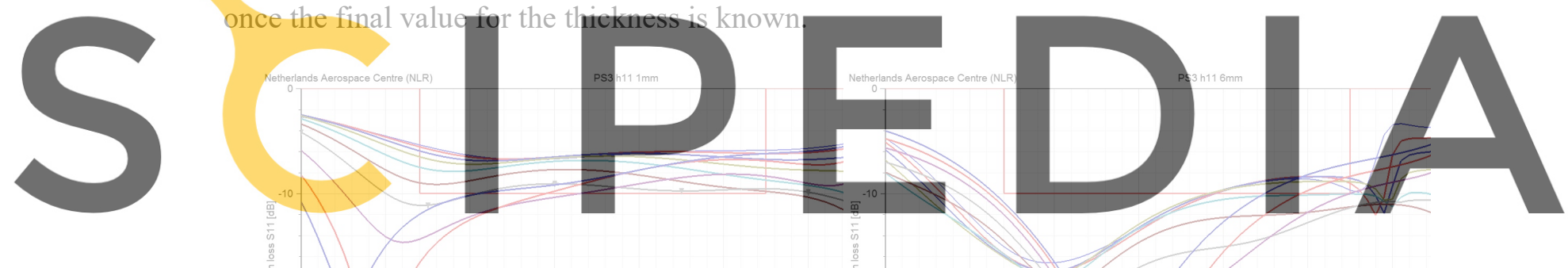

Register for free ${ }_{22}$ at bttps//www.scipedia.com to download the version without the watermark

Figure 7. Influence of thickness and distance of GFRP skin on refiection loss $\left(S_{11}\right)$ of an antenna eiement (left thickness $1 \mathrm{~mm}$ and right thickness $6 \mathrm{~mm}$ ).

\section{LIGHTNING PROTECTION}

The lightning protection for the antenna will be applied in two stages, on element level and on array level.

\subsection{Design of antenna elements with lightning protection}

The antenna elements (stacked patches) will have both patches grounded using a connecting via in the centre of the patch. In this location the grounding via will have no influence on the electromagnetic performance of the antenna element. In Figure 8 the metal parts of the antenna element are shown. The ground via in the centre of the patches is clearly visible. This via will be used to avoid static charges on the patches and to provide a safe path for lightning currents. 


\subsection{Lightning protection for the antenna array}

In addition some kind of lightning diversion needs to be added to the non-conductive GFRP skin. In general the skin has a copper mesh on the outside to conduct the lightning current. Since a copper mesh would block the electromagnetic waves, lightning diverters (strips) will applied in the GFRP skin just above the ribs of the orthogrid. These strips will be connected to the copper mesh outside the antenna window. To determine the influence of the lightning diverters, an analysis was done on a small array of $4 \times 4$ antenna elements (instead of $8 \times 8$ antenna elements). In Figure 9 the small antenna tile is shown with and without ribs and strips. For this simulation the ribs are made of perfectly conducting material (instead of CFRP with a lower conductivity).The ribs are $2 \mathrm{~mm}$ higher than the antenna tiles. The lightning strips are $4 \mathrm{~mm}$ above the antenna tile. In Figure 10 and Figure 11 the radiation patterns are shown for steering angles of 0 and 45 degrees. The influence of the lightning diverters is visible but the radiation pattern is still acceptable.
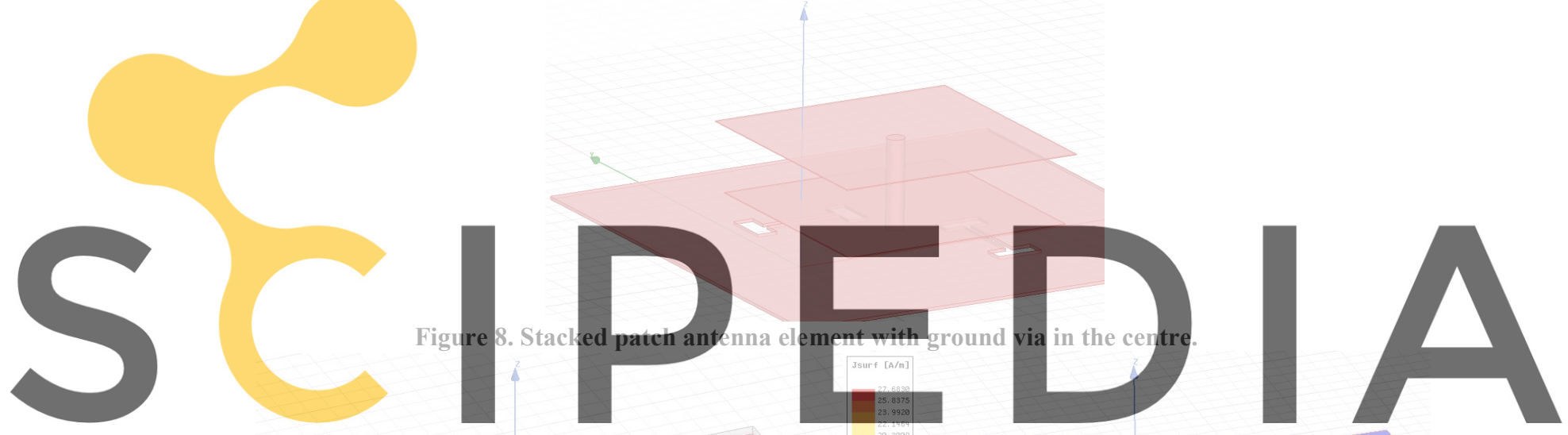

Register for free at https//www.scipedia.com to downiload the version without the watermark
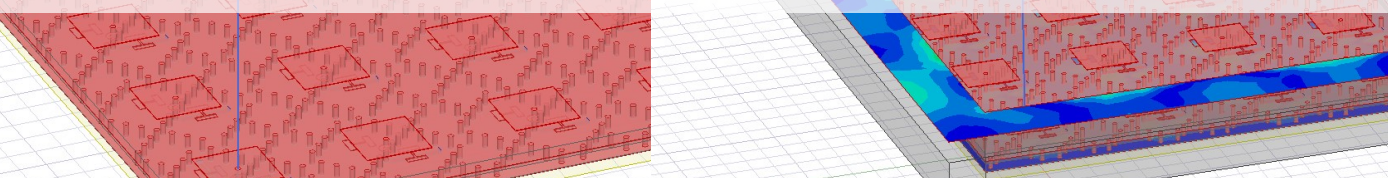

Figure 9. Tile of $4 \times 4$ antenna elements (left without ribs and strips, right with ribs and strips). \begin{tabular}{c} 
National Aerospace Centre (NLR) \\
Name Theta Ang Mag \\
\hline
\end{tabular}

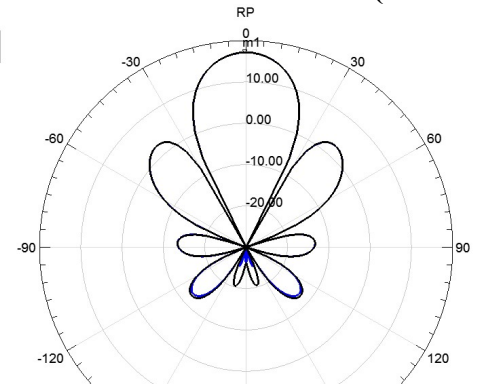

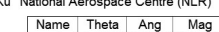
\begin{tabular}{cccc} 
Name & Theta \\
$\mathrm{m} 1$ & 0.0000 & Ang & Mag \\
\hline
\end{tabular}

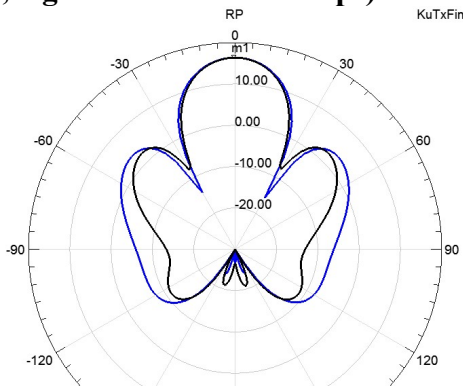

Figure 10. Influence of rib and lighting strip on radiation pattern of $4 \times 4$ array (left without ribs and strips, right with ribs and strip). Steering angle 0 degrees, two orthogonal polarisations given. 

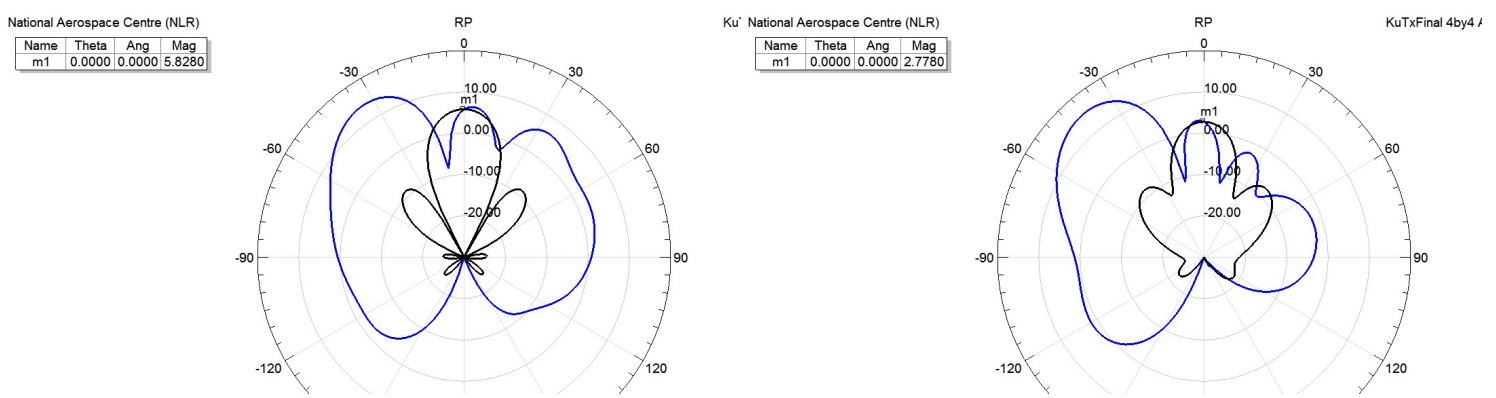

Figure 11. Influence of rib and lighting strip on radiation pattern of $4 \times 4$ array (left without ribs and strips, right with ribs and strip). Steering angle 45 degrees, two orthogonal polarisations given.

\section{CONCLUSIONS}

In this paper the structural integration of a Ku-band SATCOM antenna in the fuselage of an aircraft was addressed. In particular the specific electromagnetic aspects of the antenna integration like the lightning protection of the antenna tiles and the electromagnetic interaction of the CFRP orthogrid and GFRP fuselage skin with the array antenna were discussed. The analyses show that the separation of the antenna tiles by the ribs and the proximity of conducting materials like the ribs and the lightning diverters do have an
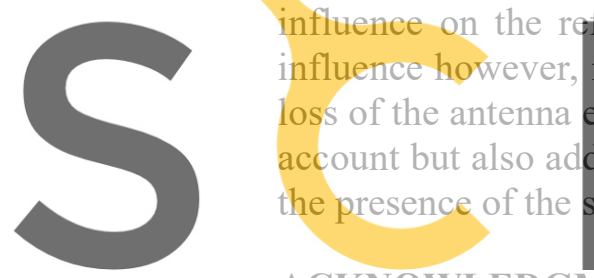

\section{ACKNOWLEDGMENT}
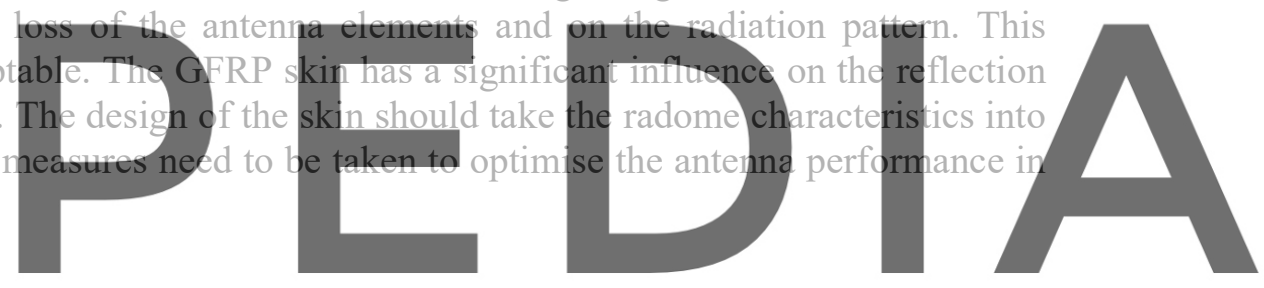

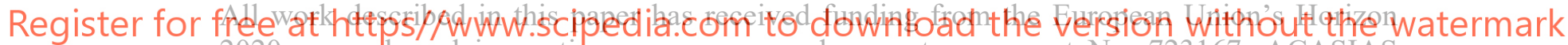
2020 research and innovation programme under grant agreement No. 723167, ACASIAS project. The authors want to acknowledge the contribution of all the partners in the ACASIAS project.

\section{REFERENCES}

[1] "Structural Analyses of Orthogrid Fuselage Panel for Integrated Ku-band SatCom Antenna", Francesc Turon, Fermin Otero and Xavier Martinez

[2] "Automated manufacture of grid stiffened panels", Peter Nijhuis, J. Marcelo Müller

[3] "Cooling of Active Components in Structurally Integrated Phased Arrays Antennas", Jens Leiß, Thomas Ebert, Marta Martínez-Vázquez and Rens Baggen

[4] ITU recommendation S.1428-1 "Reference FSS earth-station radiation patterns for use in interference assessment involving non-GSO satellites in frequency bands between 10.7 $\mathrm{GHz}$ and $30 \mathrm{GHz} "$

[5] ETSI 302186 "Harmonised Standard for satellite mobile Aircraft Earth Stations (AESs) operating in the $11 / 12 / 14 \mathrm{GHz}$ frequency bands" 